\title{
湍流工况小型风力机翼型气动特性及稳健优化
}

\author{
唐新姿 李鹏程 彭锐涛 陆金宇宇 \\ (湘潭大学机械工程学院 湘潭 411105)
}

\begin{abstract}
摘要: 风力机翼型设计通常未考虑湍流强度影响, 气动设计与实际工况产生较大偏差, 为使得翼型设计与实际工况相匹配, 考虑随机湍流工况湍流强度大小的不确定性, 以 S809 翼型为研究对象, 分析低雷诺数下不同湍流强度对翼型 S809 升阻气动 特性、压力分布影响规律, 量化湍流不确定性对翼型气动性能的影响, 提出一种在气动优化中耦合层流分离预测的高湍流低 雷诺数小型风力机翼型优化策略, 基于非嵌入式概率配置点法、Transition SST 模型、拉丁超立方试验设计、Kriging 模型和 非支配排序遗传算法进行气动稳健优化设计。案例结果表明, 优化后翼型湍流适应性增强, 在不确定湍流强度 $T I \sim N(0.15$, $0.0375^{2}$ )工况下最大升阻比平均值提升了 $6.55 \%$, 标准差减小了 $13.49 \%$ 。该方法使翼型设计与湍流风况相匹配, 降低翼型对 不确定湍流的敏感性, 为不确定湍流工况低雷诺数翼型及小型风力机设计与应用提供重要参考。
\end{abstract}

关键词: 风力机翼型; 低雷诺数; 随机湍流; 稳健性优化

中图分类号: TK83

\section{Aerodynamic Characteristics and Robust Optimization of Small Wind Turbine Airfoil under Turbulence Condition}

\author{
TANG Xinzi LI Pengcheng PENG Ruitao LU Xinyu
}

(School of Mechanical Engineering, Xiangtan University, Xiangtan 411105)

\begin{abstract}
The influence of turbulence intensity is generally not considered in the design of wind turbine airfoil, which leads to a large deviation between the aerodynamic design and the actual working condition. In order to match the airfoil design with the actual working condition, considering the uncertainty of turbulence intensity at stochastic turbulent condition, taking airfoil S809 as the research object, the influence of different turbulence intensity on the aerodynamic characteristics and pressure distribution of the airfoil S809 is analyzed, and the influence of turbulent uncertainty on the aerodynamic performance of the airfoil is quantified. An aerodynamic optimization strategy coupled with boundary layer transition prediction was proposed for high turbulence and low Reynolds number wind turbine airfoil. Based on non-intrusive probabilistic collocation method, the Transition SST model, the Latin hypercube sampling, the Kriging model and the genetic algorithm, the multi-objective optimization of airfoil was carried out. The results show that: After optimization, the airfoil turbulence adaptability is enhanced, the average lift-to-drag ratio is improved by $6.55 \%$, and the standard deviation is reduced by $13.49 \%$ under turbulence condition $T I \sim N\left(0.15,0.0375^{2}\right)$. The proposed method matches the airfoil design with turbulence, reducing the sensitivity of airfoil to turbulence uncertainty, which provides an important reference for the design and application of low Reynolds number airfoil and small wind turbines under stochastic turbulence conditions.
\end{abstract}

Key words: wind turbine airfoil; low Reynolds number; stochastic turbulence; robust design

\section{0 前言}

小型风力发电机是分布式电力能源的重要补充 形式, 由于轮毂安装位置较低, 受大气湍流演变、

* 国家自然科学基金(51305377, 51975504)、湖南省自然科学基金 (2018JJ4082)、湖南省教育厅重点(18A077)和教育部留学回国人员科研 启动基金(教外司留[2015]1098 号)资助项目。20181215 收到初稿, 20190619 收到修改稿
风切变和地表粗粘度的影响, 常在低雷诺数、不确 定高湍流流动状态下运行 ${ }^{[1]}$ 。由于雷诺数相对较低, 小型风力机翼型边界层流分离, 升力减小, 阻力增 大, 气动性能降低 ${ }^{[2]}$; 特别在不确定湍流风的作用 下，翼型表面的流动极不稳定，气动性能随之发生 较大波动, 直接影响风力机系统稳定性。开展不确 定湍流工况风力机翼型气动性能研究对于提高风力 机系统环境适应性、稳定性及可靠性有着重要意义。

针对湍流工况下翼型气动特性, 国内外学者进 
行了相关数值模拟和试验研究。YUSIK 等 ${ }^{[3]}$ 采用大 浴模拟分析了不同湍流强度下大型风力机翼型动态 失速特性。MALDONADO 等 ${ }^{[4]}$ 在风洞试验条件下 分析了两种弦长尺度湍流对 S809 翼型空气动力学 特性的影响。LI 等 ${ }^{[5]}$ 通过风洞试验研究了不同湍流 强度对翼型升阻力系数的影响。WANG 等 ${ }^{[6]}$ 在闭式 水洞超低雷诺数工况下测试了不同湍流强度下翼型 气动特性。文献研究表明, 湍流强度对风力机翼型 的气动性能具有较大的影响, 寻求适应湍流工况的 风力机翼型成为当前急需解决的问题。在翼型优化 设计方面, 李静等 ${ }^{[7-13]}$ 用分布式粒子群算法、遗传 算法、Kriging 代理模型、动网格技术、XFOIL、 RFOIL 分别对高雷诺数航空翼型、低速层流翼型与 超临界翼型、无人机翼型进行确定性气动分析或优 化设计。为考虑不确定因素影响, 国内外学者针对 航空翼型几何不确定与稳健设计开展了较多研究。

WU 等 ${ }^{[14]}$ 应用非嵌入式混沌多项式研究了几何不确 定性对跨声速翼型气动性能的影响。邬晓敬等 ${ }^{[15]}$ 采 用混沌多项式方法分析了飞行状态不确定性对航空 翼型跨声速气动载荷的影响。董世充等 ${ }^{[16]}$ 研究了翼 型表面粗䊁度存在不确定性时翼型气动性能的变 化。刘智益等 ${ }^{[17]}$ 研究了由风力机叶片安装角的随机 误差对流场及风力机气动特性产生的影响。李焦赞 等 ${ }^{[18]}$ 采用蒙特卡洛方法和 RBF 神经网络代理模型、 多目标遗传算法, 针对航空翼型几何外形制造偏差 的不确定性, 进行了几何稳健优化。DODSON 等 ${ }^{[19]}$ 采用非嵌入式混沌多项式方法研究了前缘厚度不确 定条件下翼型的稳健设计。赵轫等 ${ }^{[20]}$ 采用混沌多项 式方法研究了不确定来流马赫数条件下航空翼型稳 健设计。综上所述, 当前翼型优化研究多集中在确 定性单一工况或多工况气动研究, 稳健设计多集中 在几何不确定性方面, 而考虑不确定气动条件特别 是不确定湍流工况的风力机翼型稳健优化设计较为 鲜见。

为获得适应随机湍流工况的小型风力机翼型, 考虑湍流工况来流不确定性, 以翼型 S809 为研究对 象, 基于非嵌入式概率配置点法、Transition SST 模 型、拉丁超立方试验设计、Kriging 模型, 在气动优 化中耦合层流分离预测, 采用非支配排序遗传算法 NSGA-II 进行翼型稳健性优化设计, 该方法将翼型 设计与实际湍流风况相匹配, 为风力机及翼型设计 与应用提供参考。

\section{1 研究对象与工况}

大气边界层中随地表粗糙高度的不同, 湍流强
度一般在 $10 \% \sim 20 \%$ ，且平均湍流强度约为 $15 \%$ 。 我国风场数据统计表明, 小型大陆风场平均风速低 于 $6 \mathrm{~m} / \mathrm{s}$ 。设置湍流强度服从正态分布 $T I \sim N(0.15$, $0.0375^{2}$ ), 湍流标准差为湍流均值的 $25 \%$ 。此时风 速 $6 \mathrm{~m} / \mathrm{s}$ 湍流强度安全等级 $\mathrm{C}$ 条件下的湍流概率大 于 $90 \%$, 满足 IEC 规定的 C 级标准。S809 翼型是 美国 NREL 设计的风力机专用翼型，该翼型在小型 风力机典型雷诺数 $R e=3 \times 10^{5}$ 条件下, 最大升阻比所 在的最佳设计攻角为 $6^{\circ}$ 。为适应湍流工况, 获得 与实际湍流风况相匹配的优化翼型, 以 S809 翼型为 研究对象, 在雷诺数为 $R e=3 \times 10^{5}$ 、湍流强度为 $T I \sim$ $N\left(0.15,0.0375^{2}\right)$ 、攻角为 $6^{\circ}$ 设计工况进行不确定 分析和翼型气动稳健优化。

\section{2 研究方法}

\section{1 数值计算方法}

风力机叶片设计翼型气动数据通常在湍流强度 小于 $0.2 \%$ 的风洞测试中获得。低风速高湍流强度风 洞试验数据非常少见，使得叶片翼型设计工况与实 际工况气动特性存在较大偏差。为减少气动偏差, 考虑湍流不确定性, 在叶片设计阶段翼型数值模拟 分析中加入湍流影响。翼型数值模拟方法主要有考 虑位势流和边界层黏性-无黏迭代方法、基于 $N$-S 方 程的计算流体力学方法等。以 XFOIL ${ }^{[10]} 、 \mathrm{RFOIL}^{[13]}$ 为代表的位势流和边界层黏性-无黏迭代方法, 可以 在高雷诺数下快速地获得较好精度的气动参数。而 在低雷诺数下, 翼型边界层产生层流分离泡, 湍流 更容易改变其流动状态，影响数值模拟准确性的核 心问题在于边界层转捩和层流分离泡的准确预测。

为获得准确、可靠的气动数据, 采用修正转捩 Transition SST 模型求解 $N-S$ 方程, 该模型在 SST $k-\omega$ 基础上增加考虑分离流动的修正间歇因子以及与湍 流转捩准则相关的输运方程

$$
\begin{gathered}
\frac{\partial(\rho k)}{\partial t}+\frac{\partial\left(\rho u_{j} k\right)}{\partial x_{j}}=\frac{\partial}{\partial x_{j}}\left[\left(\mu+\sigma_{k} \mu_{t}\right) \frac{\partial k}{\partial x_{j}}\right]+\tilde{P}_{k}-\tilde{D}_{k} \\
\left\{\begin{array}{l}
\tilde{P}_{k}=\gamma_{\text {eff }} P_{k} \\
\tilde{D}_{k}=\min \left(\max \left(\gamma_{\text {eff }}, 0.1\right), 1\right) D_{k}
\end{array}\right. \\
\left\{\begin{array}{l}
\gamma_{\text {eff }}=\max \left(\gamma, \gamma_{\text {sep }}\right) \\
\gamma_{\text {sep }}=2 F_{\theta t} \cdot \min \left(1.0, F_{\text {reattach }} R e_{v \max }\right)
\end{array}\right. \\
\left\{\begin{array}{l}
R e_{v \max }=\max \left(0, \frac{R e_{v}}{3.235 R e_{\theta c}}-1.0\right) \\
F_{\text {reattach }}=\exp \left[-\left(\frac{R_{T}}{20}\right)^{4}\right]
\end{array}\right.
\end{gathered}
$$


式中, $P_{k} 、 D_{k}$ 分别是原 SST 湍流方程的生成项和耗 散项。 $\rho$ 为密度, $\mathrm{kg} / \mathrm{m}^{3} ; t$ 为时间; $u_{j}$ 为速度; $j$ 为 张量中的自由标; $x_{j}$ 为坐标值; $\theta$ 为动量厚度; $\mu$ 为 层流黏性系数; $\mu_{\mathrm{t}}$ 为湍流黏性系数。

计算流域为二维 $\mathrm{C}$ 型, 流域边界及网格, 如 图 1 所示。流域长度和宽度分别为 $35 c$ 和 $30 c$ ( $c$ 为 弦长), 翼型后方长度为 $20 c$ 。速度入口指定来流速 度、方向; 流域右边界为压力出口; 翼面为无滑移 壁面条件。近壁面第一层网格高度为 $10^{-5} \mathrm{c}$, 法向增 长率为 1.1 , 进行局部加密, 满足 $y^{+}$小于 1 。经过网 格无关性研究, 最终网格总数为 89700 。基于压力 算法和二阶迎风差分格式, SIMPLE 算法求解, 各 残差收敛小于 $1 \times 10^{-5}$ 。

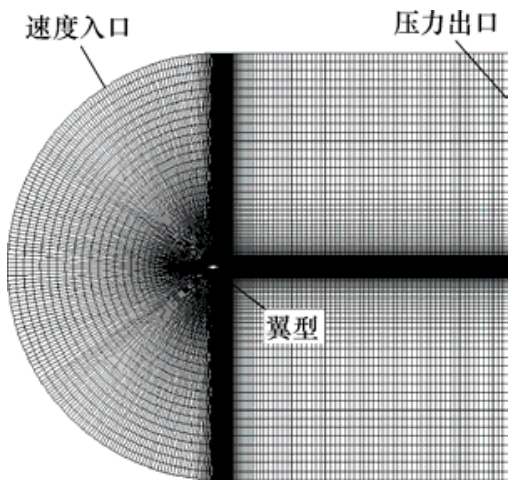

图 1 流域边界及网格

图 2 为 $6^{\circ}$ 攻角下采用修正转捩 Transition SST 模型与中国科学院工程热物理研究所(简称 IET)试 验 ${ }^{[21]}$ 所得到的压力分布。由图可见, 压力系数计算 值与 IET 试验值吻合较好, 表明所采用的计算方法 低雷诺数流动适用性较好。

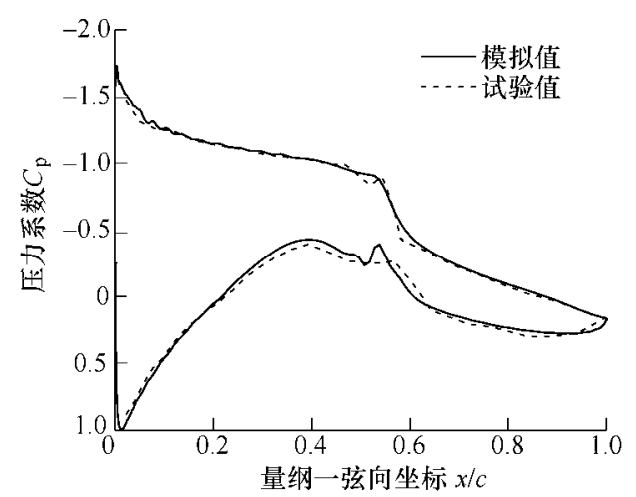

图 2 Transition SST 模型与 IEC 试验的压力分布对比

\section{2 非嵌入式概率配置点法}

常用不确定分析方法主要有蒙特卡洛方法和混 沌多项式方法 ${ }^{[20]}$ 等。其中, 蒙特卡洛方法通常需要 较大的样本点, 在数值模拟的基础上直接耦合稳健 优化将需要强大的计算资源和耗费大量计算时间, 导致设计效率低、应用难度大; 混沌多项式方法对
随机变量进行混沌多项式展开, 将其嵌入到系统模 型内部进行求解得到随机响应系数, 求解过程复杂 繁琐。非嵌入式概率配置点法将连续随机变量转化 为有限随机变量点, 降低问题维度, 通过少数随机 变量点的值来进行不确定性分析, 是多项式混沌法 与配置点法的结合。该方法将任意样本空间 $\Omega$ 上的 变量 $u(x, t, \theta)$ 展开表示为 ${ }^{[22]}$

$$
u=\sum_{k=1}^{N} u_{k}(x, t) h_{k}(\xi(\theta))
$$

式中, $u_{k}(x, t)$ 是变量 $u_{k}(x, t, \theta)$ 在第 $k$ 个配置点 $\theta_{k}$ 的值, $N$ 为配置点的个数, $h_{k}$ 表示与该点对应的 拉格朗日插值多项式混沌， $\xi(\cdot)$ 为随机变量。用 一个广义随机微分方程, 可以表示为

$$
L(a(\theta)) u(\boldsymbol{x}, t, \theta)=S(\boldsymbol{x}, t)
$$

式中, 与自由变量 $a(\theta)$ 相关的 $L(a(\theta))$ 是包含空间 和时间的微分算子, $S(x, t)$ 是与空间和时间相关的 源项。

该方法利用高斯积分点获取配置点, 高斯积分 点通过求取 Gauss-Hermite 求积公式的求积节点得 到。将选取的高斯积分点及对应的求积系数映射到 随机空间 $\Omega$ 上，即得到配置点及其权重。

$$
\begin{gathered}
u_{k}=\sqrt{2} x_{k} \sigma+\mu \\
\omega_{k}=H_{k} / \sqrt{\pi}
\end{gathered}
$$

式中, $x_{k}$ 为 Gauss-Hermite 高斯积分点, $H_{k}$ 为 Gauss-Hermite 求积系数。 $u_{k}$ 为在第 $k$ 个配置点上确 定性计算得到， $\omega_{k}$ 为其对应的权重。

表 1 给出了湍流强度服从 $T I \sim N\left(0.15,0.0375^{2}\right)$ 正态分布时对应的配置点和权重。

\section{表 1 二阶和三阶配置点与对应权重}

\begin{tabular}{cccccc}
\hline & & & \multicolumn{2}{c}{ 三阶 } \\
\cline { 1 - 2 } \cline { 5 - 6 } 配置点 $u_{k}$ & 权重 $\omega_{k}$ & & 配置点 $u_{k}$ & 权重 $\omega_{k}$ \\
\hline 0.085048 & 0.166667 & & 0.062459 & 0.045876 \\
0.15 & 0.666667 & & 0.122176 & 0.454124 \\
0.214952 & 0.166667 & & 0.177824 & 0.454124 \\
- & - & & 0.237541 & 0.045876 \\
\hline
\end{tabular}

在得到非嵌入式概率配点法展开式中的各个系 数后, 变量的均值、方差可以表示为

$$
\begin{gathered}
u_{u}=\sum_{k=1}^{N} \omega_{k} u_{k}(\boldsymbol{x}, t) \\
\sigma_{u}{ }^{2}=\sum_{k=1}^{N} \omega_{k} u_{k}{ }^{2}(\boldsymbol{x}, t)-u_{u}{ }^{2}
\end{gathered}
$$

图 3 给出了采用二阶和三阶配置点法计算的 
翼型表面压力系数的均值及标准差。由图可见, 在攻角 $6^{\circ}$ 下的二阶和三阶压力系数的均值和标 准差结果几乎重合, 验证了采用二阶配置方法的 收敛性。

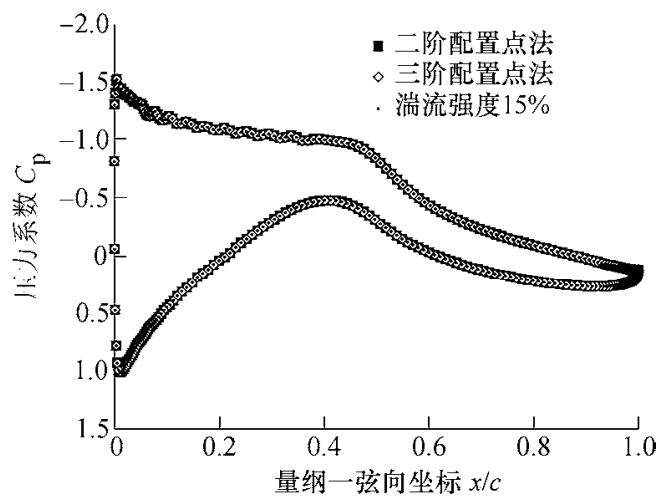

(a) 翼型表面压力分布均值及确定值

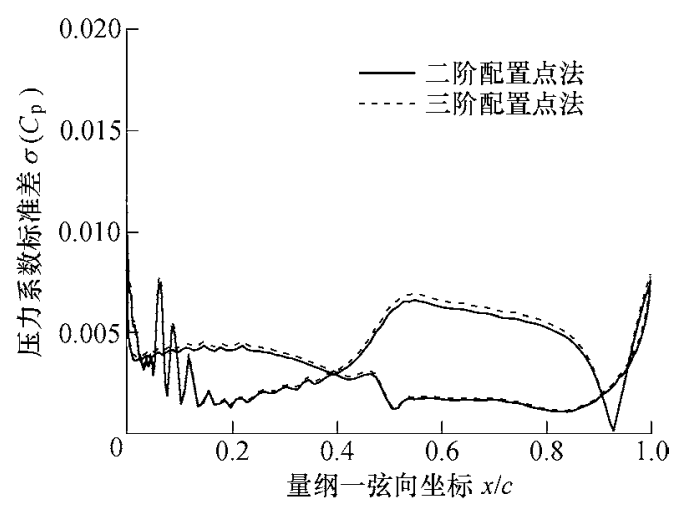

(b) 翼型表面压力标准差分布

图 3 翼型表面压力分布

\section{3 翼型几何参数化}

Hicks-Henne 型函数设计变量少, 精度高且稳定 性强, 但对翼型尾缘控制能力较差。改进的 HicksHenne 型函数 ${ }^{[23]}$ 改善了翼型尾缘夹角表征, 其表达 式可描述为

$$
\begin{gathered}
y_{\mathrm{u}}(x)=y_{0 \mathrm{u}}(x)+\sum_{k=1}^{k=n} c_{k} f_{k}(x) \\
y_{1}(x)=y_{01}(x)+\sum_{k=1}^{k=n} c_{k+n} f_{k}(x) \\
\quad(x)= \begin{cases}x^{0.25}(1-x) \exp (-20 x) & k=1 \\
\sin ^{3}(\pi x \exp (k)) & 2 \leqslant k \leqslant n-1(1) \\
\alpha x(1-x) \exp (-\beta(1-x)) & k=n \\
\ln x_{k} & 0 \leqslant x_{k} \leqslant 1\end{cases}
\end{gathered}
$$

式中, 下标 $\mathrm{u}$ 和 $\mathrm{l}$ 分别表示上下翼面, 0 表示基准翼 型; $n$ 表示数量, 主要是以相关要求获取; $c_{k}$ 为各型 函数对应系数。图 4 给出了采用改进的 Hicks-Henne 型函数对 S809 翼型在设计变量范围内进行表征。

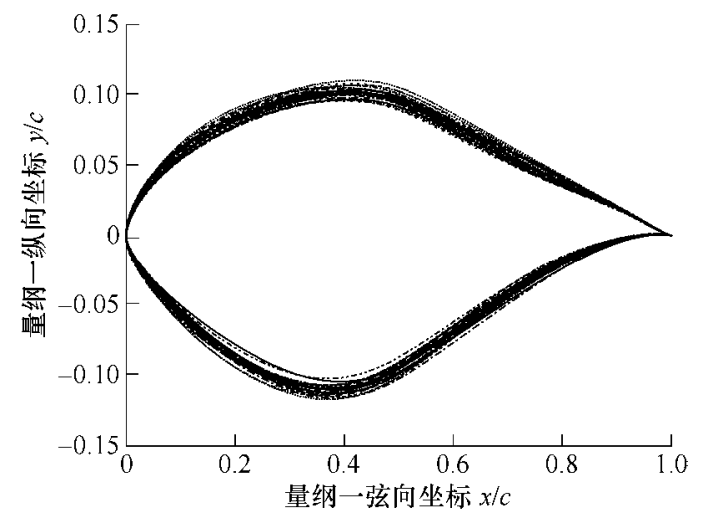

图 4 翼型参数化表征

\section{4 试验设计}

拉丁超立方抽样方法(Latin hypercube sampling, LHS) ${ }^{[24]}$ 用较少的样本点获得较全面的空间信息, 抽 取样本点均匀, 不用考虑问题的维数, 降低了试验 的规模。采用 LHS 进行抽样, 样本点数目为 150 组。

\section{5 代理模型}

为获得高效可靠的非线性多峰值拟合, 建立 Kriging 代理模型。系统响应值与自变量之间的关系 表示为 ${ }^{[25-26]}$

$$
\left\{\begin{array}{l}
y(x)=F(\beta, x)+z(x) \\
F(\beta, x)=\sum_{k=1}^{k=p} f_{k}(x) \beta_{k}
\end{array}\right.
$$

式中, $y(x)$ 为未知 Kriging 模型, $F(\beta, x)$ 为回归模型, 有 $P$ 个已知函数组成, 是一个确定性部分, $\beta$ 为回 归模型参数，其协方差可表示为

$$
\operatorname{cov}\left[Z\left(x^{i}\right), Z\left(x^{j}\right)\right]=\sigma^{2} \boldsymbol{R}\left[\boldsymbol{R}\left(x^{i}, x^{j}\right)\right]
$$

式中, $\boldsymbol{R}$ 是相关矩阵, $\boldsymbol{R}\left(x^{i}, x^{j}\right)$ 表示任意 2 个样本点 $x^{i}$ 和 $x^{j}$ 的相关函数, 选取高斯函数作为相关函数, 其表达式为

$$
\boldsymbol{R}\left(x^{i}, x^{j}\right)=\exp \left[-\sum_{k=1}^{m} \theta_{k}\left|x_{k}^{i}-x^{j}\right|^{2}\right]
$$

式中, $m$ 为设计变量的个数, $\theta_{k}$ 为模型参数。误差 平方 $R^{2}$ 衡量代理模型精度, 可以表示为

$$
R^{2}=1-\frac{\sum_{i=1}^{m}\left(y_{i}-\hat{y}_{i}\right)^{2}}{\sum_{i=1}^{m}\left(y_{i}-\bar{y}\right)^{2}}
$$

式中, $m$ 为测试样本点个数, $y_{i}$ 为试验值, $\hat{y}_{i}$ 为代 理模型的预估值, $\bar{y}$ 为试验点集的均值。

采用 CFD 方法对 130 组样本点进行计算, 并构 建 Kriging 代理模型, 20 组样本点验证精度, 计算 所得升阻比 $C_{\mathrm{l}} / C_{\mathrm{d}}$ 误差平方分别为 $0.9444 、 0.9384$ 、 0.9353 , 满足精度要求。 


\section{6 稳健设计流程}

图 5 为随机湍流低雷诺数翼型气动稳健优化设 计流程。如图 5 所示, 首先选取改进的 Hicks-Henne 参数化方法对基准翼型进行参数化, 确定设计变量 及其设计空间, 同时确定湍流度的分布范围, 然后 采用拉丁超立方法在设计空间内抽取几何外形样本 点, 并通过非嵌入式概率配点法对湍流强度进行配 置。通过改进的 Hicks-Henne 型函数对翼型进行参 数化表征, 再应用 CFD 方法求解翼型样本响应值。 根据样本数据库建立 Kriging 代理模型, 最后利用 非支配排序遗传算法进行多目标气动寻优。

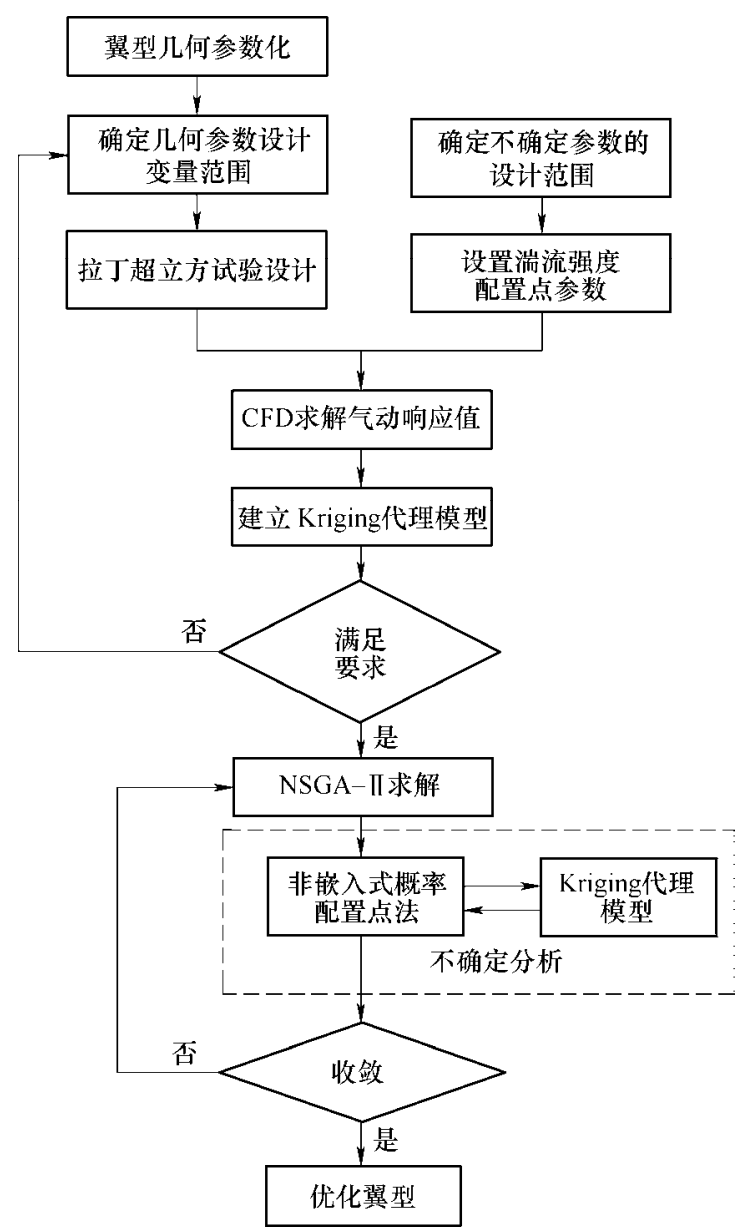

图 5 翼型稳健性优化设计流程

\section{3 不确定湍流对翼型气动性能的影响}

\section{1 升阻特性}

为量化不确定湍流对翼型最大升阻比的影响程 度并分析影响机理, 采用数值计算方法和非嵌入式 概率配置点法对不确定湍流强度下翼型最佳升阻比 攻角进行气动性能计算。表 2 给出了 $6^{\circ}$ 攻角三种 不同湍流强度下 S809 翼型升阻特性计算值。由表可 以看出, 湍流强度越大, 升阻比越小, 湍流强度越 小，气动性能越好。湍流强度由 0.0850 到 0.2150
时,升力系数由 0.7071 增加到了 0.7206 , 提升了 $1.91 \%$, 阻力系数由 0.0223 增加到了 0.0297 , 上升 了 $33.18 \%$, 升阻比由 31.7076 减小到了 24.2460 , 下降了 23.53\%。根据非嵌入式概率配点法计算可 得, 升力系数的标准差为 0.0045 , 占均值 $0.63 \%$, 阻力系数的标准差为 0.002 , 占均值 $8.04 \%$, 升阻 比标准差为 2.190 , 占均值 $8.00 \%$ 。以上结果说明, 湍流强度对升阻比有较大影响, 且对阻力系数影响 相对较大。在不确定湍流强度 $T I \sim N\left(0.15,0.0375^{2}\right)$ 下, 升阻比不确定性达到 8.00\%。

表 $26^{\circ}$ 攻角不同湍流强度下 $\mathrm{S809}$ 翼型升阻特性 $\left(R e=3 \times 10^{5}\right)$

\begin{tabular}{cccc}
\hline 湍流强度 $T I$ & 升力系数 $C_{l}$ & 阻力系数 $C_{d}$ & 升阻比 $C_{/} / C_{d}$ \\
\hline 0.0850 & 0.7071 & 0.0223 & 31.70762 \\
0.1500 & 0.7090 & 0.0261 & 27.12242 \\
0.2150 & 0.7206 & 0.0297 & 24.24596 \\
\hline
\end{tabular}

\section{2 摩阻分布}

图 6a 为上翼面不同湍流强度下的摩阻系数。由 图 6a 可以看出, 随着湍流强度的增加, 翼型表面的 摩阻系数也有所增加。当湍流强度发生变化时, 阻 力系数会随着摩阻系数的变化而变化, 阻力系数对 湍流强度的不确定性较为敏感。图 $6 \mathrm{~b}$ 为上翼面摩阻 系数的标准差沿翼型的分布。由图可以看出, 摩阻 系数最大标准差达到 0.0012 , 为均值 $11.60 \%$; 且 摩阻系数标准差从前缘到尾缘逐渐减小, 在翼型中

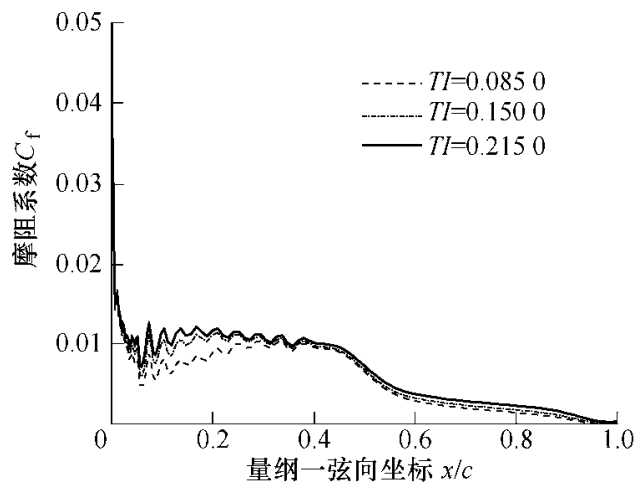

(a) 不同湍流强度下上翼面摩阻系数分布

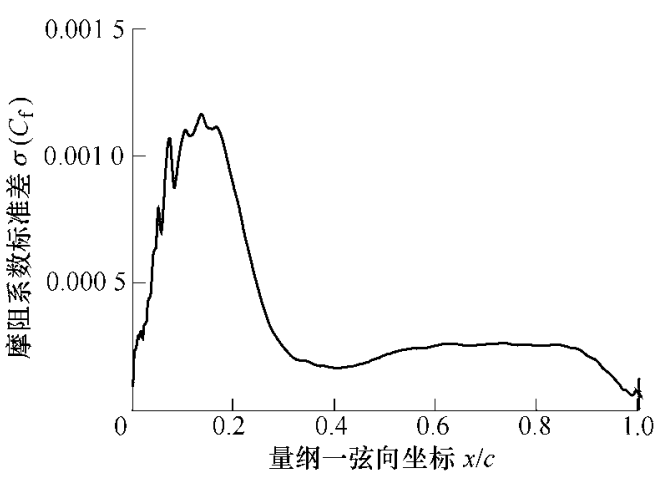

(b) 摩阻系数标准差沿上翼面的分布

图 6 摩阻系数分布 
部有所增加, 前缘最大, 表明翼型前缘对湍流强度 的不确定性较为敏感。

\section{3 压力分布}

图 7a 为上下翼面不同湍流强度下压力分布。由 图可以看出, 三种湍流强度下, 压力系数基本重合, 表明湍流强度对压力系数的影响较小。图 7b 为不确 定湍流强度下压力系数标准差。由图可以看出, 上 翼面压力系数标准差前缘波动明显, 中部和尾部幅 值相对平稳, 表明翼型上翼面前缘对不确定湍流较 为敏感。

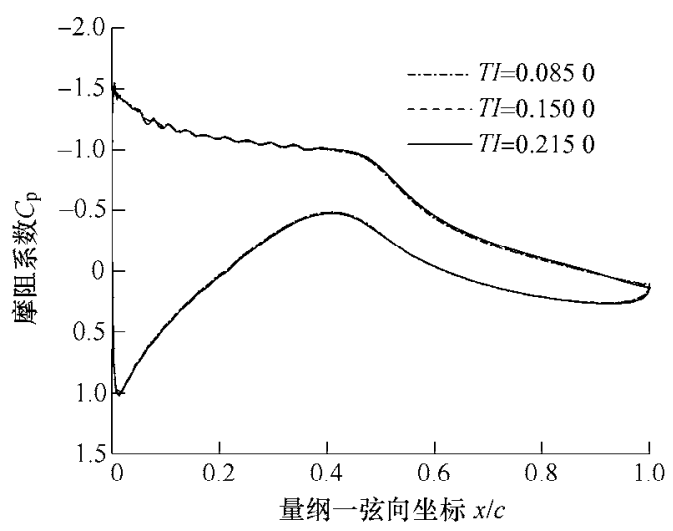

(a) 不同湍流强度下上翼面压力系数分布

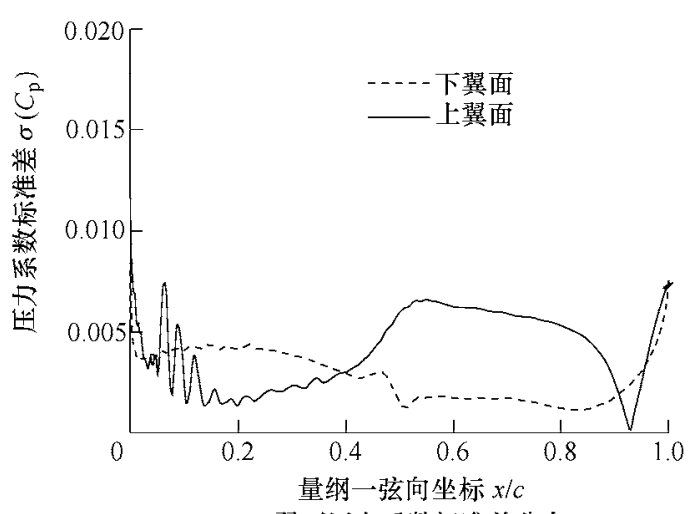

(b) 翼面压力系数标准差分布

图 7 压力系数分布

\section{4 翼型气动稳健优化}

\section{1 优化数学模型与求解}

以基准翼型 $\mathrm{S} 809$, 雷诺数 $R e=3 \times 10^{5}$, 攻角 $\alpha=6^{\circ}$ 为例, 进行稳健性优化, 以获得在湍流工况下升阻 比较高且性能稳定的优化翼型。湍流强度服从正态 分布 $T I \sim N\left(0.15,0.0375^{2}\right)$, 采用非嵌入式概率配置 点法进行配置。根据前面分析可知不确定性对翼型 前缘的影响较大, 调整控制点参数范围, 避免交叉、 不光顺的现象。设计变量为翼型表征型函数系数 $c_{k}$, 取值范围如表 3 所示。
表 3 设计变量取值范围

\begin{tabular}{ccc||ccc}
\hline 设计变量 & 下限 & 上限 & 设计变量 & 下限 & 上限 \\
\hline$C_{1}$ & -0.008 & 0.008 & $C_{7}$ & -0.008 & 0.008 \\
$C_{2}$ & -0.007 & 0.007 & $C_{8}$ & -0.007 & 0.007 \\
$C_{3}$ & -0.006 & 0.006 & $C_{9}$ & -0.006 & 0.006 \\
$C_{4}$ & -0.005 & 0.007 & $C_{10}$ & -0.007 & 0.005 \\
$C_{5}$ & -0.005 & 0.003 & $C_{11}$ & -0.003 & 0.005 \\
$C_{6}$ & -0.004 & 0.002 & $C_{12}$ & -0.002 & 0.004 \\
\hline
\end{tabular}

优化数学模型为

$$
\begin{gathered}
\text { Object: }\left\{\begin{array}{l}
\operatorname{maxmize} \mu\left(C_{l} / C_{d}\right) \\
\operatorname{minmize} \sigma\left(C_{l} / C_{d}\right)
\end{array}\right. \\
\text { s.t. }\left\{\begin{array}{l}
X_{\text {tr_up }}>X_{\text {tr_up0 }} \\
|t-0.21|<0.02 \\
\left|X_{\mathrm{t}, \text { max }}-0.395\right|<0.03 \\
C_{k} \in\left[\min _{k}, \text { max }_{k}\right]
\end{array}\right.
\end{gathered}
$$

式中, 下标 0 代表基准翼型, 下标 $n$ 表示优化迭代

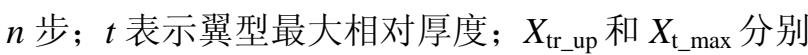
表示表示上翼面转捩位置和最大相对厚度位置。 min、 $m a x$ 分别为设计变量范围的上下限。设置初始 群体规模 200, 进化代数 100, 交叉概率 0.9 , 变异 概率 0.01 。

\section{5 优化结果与讨论}

\section{1 优化前后翼型几何对比}

图 8 为求解寻优后得到的 Pareto 解集前沿。选 取三个优化翼型样本，并与基准翼型的性能进行对 比。由图 8 可知, 优化翼型的升阻比均值明显提升, 标准差下降。

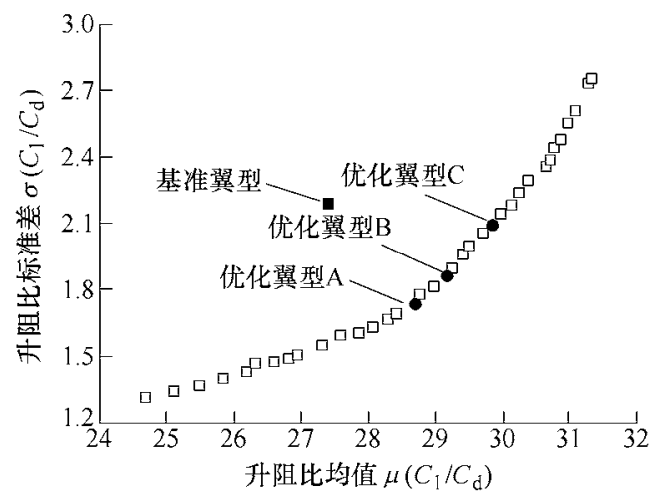

图 8 Pareto 前沿

优化翼型和基准翼型升阻比均值和标准差对比 如表 4 所示。由表 4 可知, 优化翼型 $\mathrm{A}$ 最大升阻比 均值提高 4.74\%, 标准差下降 20.80\%; 优化翼型 B 最大升阻比均值提高 $6.47 \%$, 标准差下降 $14.93 \%$; 
优化翼型 C 最大升阻比均值提高 8.94\%, 标准差下 降 4.41\%。将所得三种优化翼型进行 CFD 计算验证, 并结合配置点法得到升阻比均值和标准差, CFD 计 算结果与优化结果基本吻合。

表 4 基准翼型和优化翼型升阻比对比

\begin{tabular}{ccccc}
\hline & $\begin{array}{c}\text { 升阻比 } \\
\text { 均值 }\end{array}$ & $\begin{array}{c}\text { 相对变化 } \\
(\%)\end{array}$ & $\begin{array}{c}\text { 升阻比 } \\
\text { 标准差 }\end{array}$ & $\begin{array}{c}\text { 相对变化 } \\
(\%)\end{array}$ \\
\hline 基准翼型 & 27.4032 & - & 2.1909 & - \\
优化翼型 A & 28.7014 & 4.74 & 1.7352 & -20.80 \\
优化翼型 B & 29.1754 & 6.47 & 1.8637 & -14.93 \\
优化翼型 C & 29.8519 & 8.94 & 2.0942 & -4.41 \\
\hline
\end{tabular}

综合考虑风力机翼型的性能与稳健性, 选择优 化翼型 $\mathrm{B}$ 作进一步对比分析。图 9 为优化翼型 $\mathrm{B}$ 和 基准翼型的几何外形对比。优化后翼型的前缘半径 减小, 最大厚度位置后移。最大相对厚度由 $21.00 \% c$ 减小至 $19.80 \% c$, 最大厚度位置由 $39.50 \% c$ 移至 $39.54 \% c$ 。

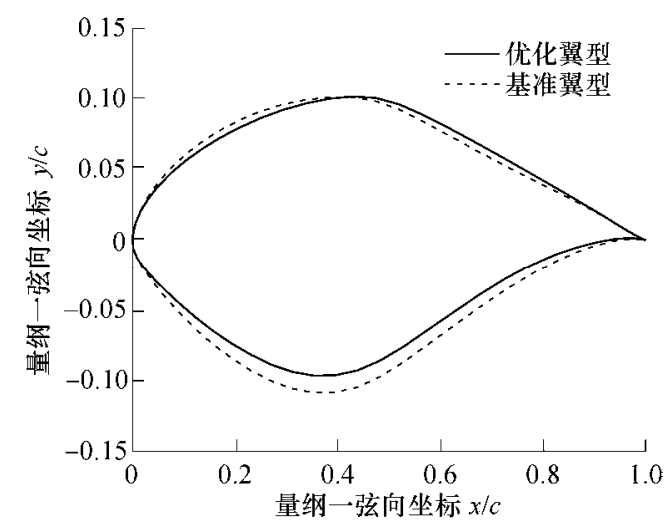

图 9 基准翼型和优化翼型几何外形对比

\section{2 优化前后翼型升阻特性对比}

图 10 给出了不同湍流强度下基准翼型和优化 翼型升阻特性对比。与基准翼型相比，在不同的湍 流工况下, 优化翼型升阻比均得以提升, 气动性能 得到改善。与基准翼型相比, 湍流强度为 0.15 时, 优化翼型升阻比由 27.1183 增加到 29.094 , 提升 $7.29 \%$ 。

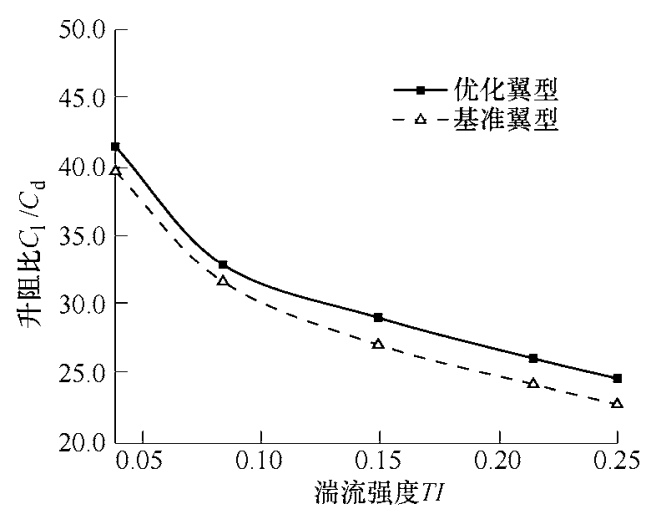

图 10 不同湍流强度下基准翼型和优化翼型升阻特性对比
表 5 给出湍流工况下基准翼型和优化翼型气 动稳健性对比。由表可知, 优化翼型的气动性能得 到改善, 并且气动稳健性得到提升。在不确定湍流 强度 $T I \sim \mathrm{N}\left(0.15,0.0375^{2}\right)$ 下，优化翼型平均升阻 比提升 $6.55 \%$, 标准差减小 $13.49 \%$, 能更好地满足 低速风力机湍流工况下对风力机叶片翼型的要求。

\section{表 5 不同湍流工况下基准翼型和优化翼型气动稳健性对比}

\begin{tabular}{lccccc}
\hline \multicolumn{1}{c}{ 湍流强度配置点 } & 0.0850 & 0.1500 & 0.2150 & 均值 & 标准差 \\
\hline 基准裂型升阻比 & 31.7034 & 27.1183 & 24.2427 & 27.4032 & 2.1909 \\
优化翼型升阻比 & 32.6801 & 29.0952 & 26.1336 & 29.1991 & 1.8953 \\
优化前后相对变化(\%) & 3.08 & 7.29 & 7.80 & 6.55 & -13.49 \\
\hline
\end{tabular}

\section{6 结论}

(1) 采用非嵌入式概率配置点法进行不确定分 析, 量化了随机湍流对翼型的气动性能的影响程度。 结果表明, 翼型阻力系数和升阻比对湍流强度不确 定较为敏感。通过不确定性在流场中传播分析发现 不确定湍流敏感几何部位主要位于翼型前缘。

（2）基于不确定性分析，提出了随机湍流工况 条件下风力机翼型的稳健优化设计方法。该方法将 非嵌入式概率配置点法与优化算法相结合, 通过少 数随机变量点进行不确定分析, 将连续的随机变量 转化为有限的随机变量点, 降低问题分析维度; 基 于配置点和样本库构建不确定代理模型, 为不确定 条件下风力机翼型稳健性设计提供了新的思路。

(3) 考虑不确定湍流条件的影响, 降低了翼型 对不确定湍流度的影响, 使翼型设计与湍流风况相 匹配, 风力机翼型湍流风况适应性增强。

\section{参 考 文 献}

[1] 李鸿秀, 朱瑞兆, 王萝, 等. 不同地形风电场湍流强度 日变化和变化分析 [J]. 太阳能学报, 2014, 35(11): 2327-2333.

LI Hongxiu, ZHU Ruizhao, WANG Rui, et al. Wind turbulence intensity of daily and yearly variation analysis in different kinds of terrain[J]. Acta Energies Solaris Sinica, 2014, 35(11): 2327-2333.

[2] 唐新姿, 黄轩晴, 孙松峰, 等. 考虑层流分离的低速风 力机翼型气动性能研究[J]. 动力工程学报, 2017,37(1): 52-59.

TANG Xinzi, HHUANG Xuanqiang, SUN Songfeng, et al. Study on aerodynamic characteristics of low-speed wind turbine airfoil in consideration of laminar separation [J]. Journal of Chinese Society of Power Engineering, 2017, 37(1): 52-59. 
[3] YUSIK K, XIE Z T. Modelling the effect of freestream turbulence on dynamic stall of wind turbine blades[J]. Computers and Fluids, 2016, 12(9): 53-56.

[4] MALDONADO V, CASTILLO L, THORMANNC A, et al. The Role of free stream turbulence with large integral scale on the aerodynamic performance of an experimental low Reynolds number S809 wind turbine blade[J]. Journal of Wind Engineering and Industrial Aerodynamics, 2015, 142: 246-257.

[5] LI Q, KAMADA Y, MAEDA T, et al. Effect of turbulent inflows on airfoil performance for a horizontal axis wind turbine at low Reynolds numbers (part I: Static pressure measurement)[J]. Energy, 2016, 111: 701-712.

[6] WANG S, ZHOU Y, YANG H, et al. Turbulent intensity and Reynolds number effects on an airfoil at low Reynolds numbers[J]. Physics of Fluids, 2014, 26(11): 257-263.

[7] 李静, 高正红, 黄江涛, 等. 基于分布式粒子群算法的 翼型优化设计 $[\mathrm{J}]$. 空气动力学学报, 2011, 29(4): 464-469.

LI Jing, GAO Zhenghong, HUANG Jiangtao, et al. Airfoil optimization based on distributed particle swarm algorithm[J]. Acta Aerody-namica Sinica, 2011， 29(4): 464-469.

[8] 王迅, 蔡晋生, 屈芘, 等. 基于改进 CST 参数化方法 和转捩模型的翼型优化设计 [J]. 航空学报, 2015, 36(2): 449-461.

WANG Xun, CAI Jinsheng, QU Kun, et al. Airfoil optimization based on improved CST parametric method and transition model[J]. Acta Aeronautica et Astronautica Sinica, 2015, 36(2): 449-461.

[9] 朱雄峰, 郭正, 侯中喜, 等. 基于动网格的翼型设计优 化[J]. 国防科技大学学报, 2013, 35(2): 1-6.

ZHU Xiongfeng, GUO Zheng, HOU Zhongxi, et al. Dynamic mesh based airfoil design optimization[J]. Journal of National University of Defense Technology, 2013, 35(2): 1-6.

[10] 陈学孔, 郭正, 易凡, 等. 低雷诺数翼型的气动外形优 化设计[J]. 空气动力学学报, 2014, 32(3): 300-307. CHEN Xueli, GUO Zheng, YI Fan, et al. Aerodynamic shape optimization and design of airfoils with low Reynolds number[J]. Acta Aerodynamica Sinica, 2014, 32(3): 300-307.

[11] 陈进, 蒋传鸿, 谢翌, 等. 典型霜冰条件下的风力机翼 型优化设计[J]. 机械工程学报, 2014, 50(7): 154-160. CHEN Jin, JIANG Chuanhong, XIE Yi, et al. Optimization design of airfoil for wind turbine under typical rime icing conditions[J]. Journal of Mechanical Engineering, 2014, 50(7): 154-160.
[12] 王科雷, 祝小平, 周洲, 等. 基于转捩模型的低雷诺数 翼型优化设计研究[J]. 西北工业大学学报, 2015, 33(4): 580-587.

WANG Kelei, ZHU Xiaoping, ZHOU Zhou, et al. Studying optimization design of low Reynolds number airfoil using transition model[J]. Journal of North western Polytechnical University, 2015，33(4): 580-587.

[13] 汪泉, 陈进, 王君, 等. 基于连续攻角的风力机翼型整 体气动性能提高的优化设计 $[\mathrm{J}]$. 机械工程学报, 2017, 53(13): 143-149.

WANG Quan, CHEN Jin, WANG Jun, et al. Wind turbine airfoil optimal design with high whole aerodynamic performance considering continuous angle of attack[J]. Journal of Mechanical Engineering, 2017，53(13): 143149.

[14] WU X, ZHANG W, SONG S. Uncertainty quantification and sensitivity analysis of transonic aerodynamics with geometric uncertainty[J]. International Journal of Aerospace Engineering, 2017(1): 1-16.

[15] 邬晓敬, 张伟伟, 宋述芳, 等. 翼型跨声速气动特性的 不确定性及全局灵敏度分析[J]. 力学学报, 2015, 47(4): 587-595.

WU Xiaojing, ZHANG Weiwei, SONG Shufang, et al. Uncertainty quantification and global sensitivity analysis of transonic aerodynamics about airfoil[J]. Chinese Journal of Theoretical and Applied Mechanics, 2015, 47(4): 587-595.

[16] 董世充, 王晓东, 康顺. 表面粗粘度对翼型气动特性影 响的数值模拟 [J]. 工程热物理学报, 2015, 36(6): 12381241.

DONE Shichong, WANG Xiaodong, KANG Shun. Numerical simulationson the effect of surface roughness on airfoil aerodynamic performance[J]. Journal of Engineering Thermophysics, 2015, 36(6): 1238-1241.

[17] 刘智益, 王晓东, 康顺. 风轮失谐的气动力不确定性研 究[J]. 工程热物理学报, 2015，36(3): 517-521.

LIU Zhiyi， WANG Xiaodong, KANG Shun. Non-Deterministic research on aerodynamic force of mistuned wind turbine[J]. Journal of Engineering Thermophysics, 2015, 36(3): 517-521.

[18］李焦赞, 高正红. 基于几何不确定性的翼型多目标稳健 优化设计[J]. 力学学报, 2011，43(3): 611-615.

LI Jiaozan, GAO Zhenghong. Multi-objective optimization methodology for airfoil robust design under geometry uncertainty[J]. Chinese Journal of Theoretical and Applied Mechanics, 2011, 43(3): 611-615.

[19] DODSON M, PARKS G T. Robust aerodynamic design optimization using polynomial chaos[J]. Journal of Aircraft, 2015, 46(2): 635-646. 
[20] 赵辑, 高正红, 黄江涛, 等. 基于 PCE 方法的翼型不确 定性分析及稳健设计[J]. 力学学报, 2014, 46(1): 10-19. ZHAO Ke, GAO Zhenghong, HUANG Jiangtao, et al. Uncertainty quantification and robust design of airfoil based on polynomial chaos technique[J]. Chinese Journal of Theoretical and Applied Mechanics，2014， 46(1): 10-19.

[21] 黄宸武, 杨科, 廖猜猜, 等. S809 风力机专用翼型低雷 诺数气动特性实验研究 [J]. 工程热物理学报, 2014,

5(11): 2197-2201.

HUANG Chenwu, YANG Ke, LIAO Caicai, et al. Experimental study on aerodynamic characteristics of S809 dedicaed wind turbine airfoil at low Reynolds number[J]. Journal of Engineering Thermophysics, 2014, 5(11): 2197-2201.

[22] WANG X, HIRSCH C, LIU Z, et al. Uncertainty-based robust aerodynamic optimization of rotor blades[J]. International Journal for Numerical Methods in Engineering, 2013, 94(2): 111-127.

[23] 王建军, 高正红. HicksHenne 翼型参数化方法分析及改 进[J]. 航空计算技术，2010，40(4): 46-49.

WANG Jianjun, GAO Zhenghong. Analysis and improvement of hicks-henne airfoil parameterization method[J]. Aeronautical Computing Technique, 2010, 40(4): $46-49$.

[24] 许瑞飞, 宋文萍, 张坤. 转捩对风力机翼型优化设计结
果的影响研究[J]. 太阳能学报, 2011, 32(12): 17981803.

XU Ruifei, SONG Wenping, ZHANG Kun. Investigation of effect of transition on wind turbine airfoil optimization design[J]. Acta Energiae Solaris Sinica, 2011, 32(12): 1798-1803.

[25] 孙美建,詹浩. Kriging 模型在机翼气动外形优化中的应 用[J]. 空气动力学学报, 2011，29(6): 759-764.

SUN Meijian ZHAN Hao. Application of Kriging surrogate model for aerodynamic shape optimization of wing[J]. Acta Aerodynamica Sinica, 2011, 29(6): 759-764.

[26] 孙俊峰, 刘刚, 江雄, 等. 基于 Kriging 模型的旋翼翼 型优化设计研究 $[J]$. 空气动力学学报, 2013, 31(4): 437-441.

SUN Junfeng, LIU Gang, JIANG Xiong, et al. Research of rotor air foil design optimization based on the Kriging model[J]. Acta Aerodynamica Sinica, 2013, 31(4): 437-441.

作者简介: 唐新姿, 女, 1981 年出生, 博士, 副教授, 硕士研究生导师。 主要研究方向为风力发电技术。

E-mail: xinzitang@163.com

李鹏程, 男, 1993 年出生。主要研究方向为风力发电技术。

E-mail: lipengchengtt@163.com

彭锐涛(通信作者), 男, 1982 年出生, 博士, 教授, 博士研究生导师。 主要研究方向为气动设计及多学科优化、高效精密加工与传动。

E-mail: pengruitao@163.com 